\title{
Establishment and Application of Engineered NIH 3T3 Cell Line with Stable Human RAGE Expression
}

\author{
Xinyi Xiao ${ }^{1}$ Hui Chen ${ }^{1}$ Pameila Paerhati ${ }^{1}$ Meiqi Zhou ${ }^{1}$ Zhuoyi Yang ${ }^{1}$ Siyi Bai ${ }^{1}$ Yunsheng Yuan ${ }^{1}$ \\ ${ }^{1}$ Engineering Research Center of Cell \& Therapeutic Antibody, \\ Shanghai Jiao Tong University College of Pharmacy, Ministry of \\ Education, Shanghai, People's Republic of China \\ Address for correspondence Yunsheng Yuan, PhD, Engineering \\ Research Center of Cell \& Therapeutic Antibody, Shanghai Jiao Tong \\ University College of Pharmacy, Ministry of Education, 800 \\ Pharmaceut Fronts 2020;2:e23-e27. \\ Dongchuan Road, Shanghai 200240, People's Republic of China \\ (e-mail: yunsheng@sjtu.edu.cn).
}

\begin{abstract}
Keywords

- RAGE

- engineered cell line

- lentiviral

- transduction

- flow cytometry

Aim NIH3T3 cell line with expression of human receptor for advanced glycation endproducts (hRAGE) transduced with lentivirus vectors was used to analyze affinity, biological activity, and/or molecular mechanisms of molecules targeting the hRAGE pathway.

Method The DNA fragment coding for hRAGE gene was integrated into the genome of NIH3T3 cells using lentivirus transduction. Cells expressing hRAGE were selected with puromycin, and the level of hRAGE expression was analyzed by Western blot. To establish a stable cell line, colonies of hRAGE-expressing cells were generated, and the level of RAGE expression in each engineered cell line was analyzed within 20 generations. Flow cytometry assay was used to verify affinity of anti-hRAGE antibody binding to hRAGE on the surface of engineered cells. The engineered NIH3T3 cell line was applied to assess effects of anti-hRAGE blocking antibody on amyloid $\beta$-induced cells apoptosis by CCK-8 assay.

Results The engineered NIH3T3 cell line (hRAGE-NIH3T3) could stably express human RAGE. Commercial anti-RAGE polyclonal antibody could recognize and bind to human RAGE on the surface of hRAGE-NIH3T3 but not original NIH3T3 cells. In addition, hRAGE-NIH3T3 was more sensitive to RAGE pathway-dependent stimulation. Our data show that the hRAGE-NIH3T3 cell line established is an excellent tool in the study of RAGE-targeting molecules based on the cellular level, biological function, and RAGEmediated molecular mechanisms.
\end{abstract}

\section{Introduction}

Receptor for advanced glycation end-products (RAGE) is one of the members of the immunoglobulin superfamily and a multiligand cell surface receptor with structural homology to other immunoglobulin-like receptors. ${ }^{1}$ RAGE consists of an extracellular domain, a transmembrane domain, and a cytoplasmic domain. $^{2}$ The extracellular domain of RAGE includes a variable domain (V1) and two constant domains (C1 and C2), which are responsible for binding by different ligands. ${ }^{3} \mathrm{~V} 1$ and $\mathrm{C} 1$ domains are generally regarded as the main interaction motifs with various ligands. Although the cytoplasmic tail is very short, it is critical for intracellular signal transduction. ${ }^{4}$ RAGE has a variety of ligands, such as advanced glycation end-products (AGEs), ${ }^{5}$ high mobility group protein $B 1$ (HMGB1), ${ }^{6}$ amyloid $\beta$ protein $(A \beta),{ }^{7}$ and S100 family proteins. ${ }^{8}$ All these ligands interact with the extracellular domain of RAGE and activate downstream signaling pathways, including activation of extracellular regulated protein kinases (ERK 1/2) and phosphatidylinositol 3-kinase (PI3K). ${ }^{9-13}$ Activated RAGE promotes the production of reactive oxygen species and proinflammatory cytokines, and it also inhibits the expression of tumor suppressor genes. ${ }^{14,15}$ Overactivation of RAGE is associated with many diseases, including diabetes complications, atherosclerosis, neurodegenerative diseases, liver fibrosis, and tumors. ${ }^{9,16-20}$ 
Blockade of ligand/RAGE receptor interaction is a potential strategy for the treatment of these diseases, and RAGE has become a new target for drug discovery.

Several cell lines have been used in studies of RAGE ligand activities or biological function in vitro, such as neuroblastoma cells (SH-SY5Y), breast cancer cells (MCF-7), and human embryonic kidney cells (HEK293). However, RAGE expression is low in these cell lines, which were not suitable for investigation of the affinity of RAGE ligands or anti-RAGE antibodies. ${ }^{21}$ The transgenic cell lines play a critical role in processes of new drug development, such as drug screening, activity assay, and expression of recombinant protein or antibody, etc. Establishment of an engineered cell line for expression of human RAGE (hRAGE) was necessary to support new drug development for targeting RAGE. Mouse embryonic fibroblast NIH3T3 was widely used in cellular signal transduction, protein-protein interaction, and molecular activity assays because it was easy to maintain, transfect, and grow. ${ }^{22-24}$ In this study, NIH3T3 cells were used to generate transgenic cell lines for stable expression of hRAGE.

\section{Materials and Methods}

\section{Instrument}

A super clean bench (Suzhou Purification Equipment Co., Ltd., China), a cell counter (Ruiyun Biotechnology Co., Ltd.), a chemiluminescence imager (Tanon, China), a multifunctional microplate reader (Tecan, Switzerland), a flow cytometer (Beckman Coulter, United States), and protein electrophoresis gel equipment (Bio-Rad, United States).

\section{Materials}

NIH3T3 and HEK293T were purchased from Chinese Academy of Sciences Cell Bank (ACSCB), anti-hRAGE rabbit polyclonal antibody and rabbit immunoglobulin $G$ ( $\operatorname{IgG}$ ) from Shanghai Kaijing Biotechnology Company, China, pLVX-puro-humanRAGE-HA from Universal Biological System Co., Ltd., China, A 1 1-42 from Chutai Biotechnology Company, China, mouse anti-HA tag antibody from Proteintech, United States, mouse anti-GAPDH antibody from Proteintech, United States, fluorescein isothiocyanate (FITC)-conjugated goat anti-rabbit $\operatorname{IgG}$ $\left(\mathrm{H}^{+} \mathrm{L}\right)$ and HRP-conjugated rabbit anti-mouse $\operatorname{IgG}\left(\mathrm{H}^{+} \mathrm{L}\right)$ from Jackson, United States; CCK8 Kit from Dojindo, Japan, and substrate of enhanced chemiluminescence (ECL) from Merck Millipore, United States.

\section{Cell Culture}

All cells were maintained in high-glucose DMEM (Gibco, United States) supplemented with $10 \%$ fetal bovine serum (FBS; Gibco) and incubated at $37{ }^{\circ} \mathrm{C}$ with $5 \% \mathrm{CO}_{2}$ in a humidified cell incubator. For passaging, cells were dissociated using $0.25 \%$ trypsin-EDTA at 80 to $90 \%$ confluence and replanted at $1: 3$ dilutions.

\section{Package of Lentivirus and Transduction of Cells}

The procedure of lentivirus packaging and cell transducing were performed as previously described. ${ }^{25}$ The mixture of pLVX-puro-human-RAGE-HA plasmid (-Fig. 1), pVSV-G,

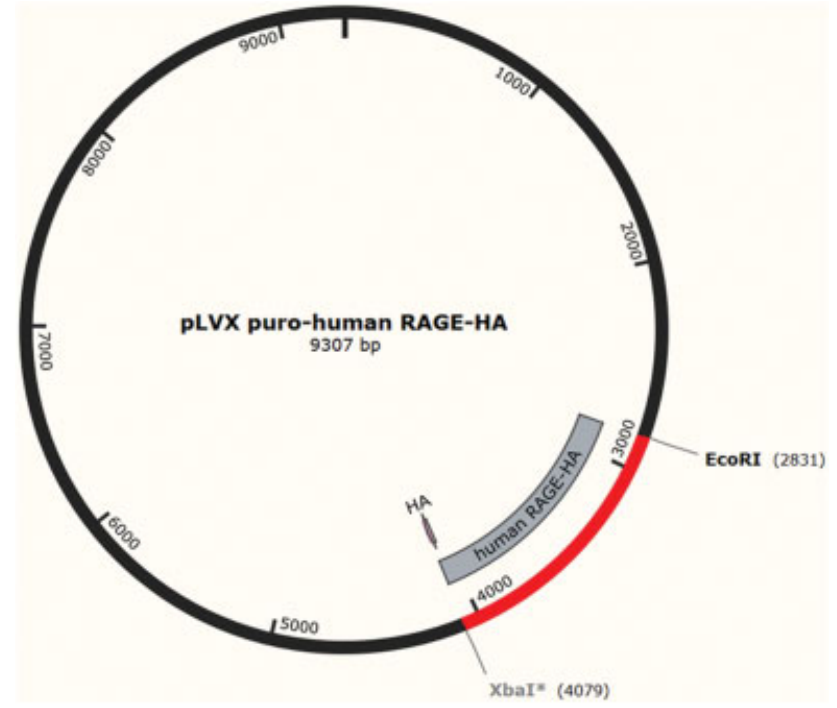

Fig. 1 The vector of pLVX-puro-human-RAGE-HA.

pRev, pTat, and pGag-pol plasmids was transfected into 293T cells by lipofectamine 2000. Lentiviral particles were harvested after 2 days of transfection. The NIH3T3 cells were seeded to a six-well plate and incubated overnight. The cells were fed with fresh medium containing lentiviral particles and polybrene whose final concentration was $10 \mu \mathrm{g} / \mathrm{mL}$. The transduced cells were selected with $10 \mu \mathrm{mol} / \mathrm{L}$ puromycin.

\section{Western Blotting}

hRAGE-NIH3T3 and NIH3T3 cells were lysed in protein lysis buffer and $\beta$-mercaptoethanol, followed by electrophoretic separation on a $10 \%$ tris-polyacrylamide gel, and then transferred onto a polyvinylidene fluoride (PVDF) membrane at $200 \mathrm{~mA}$ for 120 minutes. The membrane was blocked with $5 \%$ nonfat dry milk in TBST for 1 hour. Membranes were incubated in primary antibody (mouse anti-HA antibody, mouse antiGAPDH antibody) at $4{ }^{\circ} \mathrm{C}$ overnight and subsequently with the corresponding horseradish peroxidase-labeled secondary antibody diluted in 5\% nonfat dry milk in TBST for 45 minutes at room temperature. The signal of blot was detected by ECL substrate and captured by a Tanon Image system.

\section{Flow Cytometry Assay}

hRAGE-NIH3T3 and NIH3T3 cells were harvested and suspended by DMEM with $10 \% \mathrm{FBS}$. In total, $2.5 \times 10^{5}$ cells for each sample were centrifuged and washed three times using FACS (PBS, pH 7.2, 2\% FBS). Various concentrations of anti-hRAGE antibody $(1,2$, and $5 \mu \mathrm{g}$ ) were added to each sample, using PBS as the control. All samples were incubated for 30 minutes at $4^{\circ} \mathrm{C}$ and then washed three times with FACS. FITC-conjugated goat anti-rabbit IgG was added to the samples which were then incubated at $4^{\circ} \mathrm{C}$ in the dark. After 30 minutes, cells were washed, suspended, and analyzed by a flow cytometer.

\section{CCK8 Cell Viability Assay}

hRAGE-NIH3T3 or NIH3T3 cells were seeded in 96-well plates (5,000 cells/well) and incubated overnight in $100 \mu \mathrm{L}$ DMEM 
with $10 \%$ FBS. Then, the medium was changed and fed with DMEM with $1 \%$ FBS for starvation. After 24 hours of starvation, the cells were fed a complete medium (DMEM $+10 \%$ FBS) and treated with $A \beta(8 \mu \mathrm{mol} / \mathrm{L})$ with different concentrations of antihRAGE antibody $(3.125,6.25,12.50,25.00$, and $50.00 \mu \mathrm{g} / \mathrm{mL})$ or PBS as control. After 48 hours of treatment, cell viability was assessed by a CCK8 kit according to the manufacturer's instructions.

\section{Statistical Analyses}

Statistical analyses were performed using Prism 7.0 (GraphPad Software, San Diego, California, United States). Significant differences $(p<0.05)$ were calculated by one-way analysis of variance followed by Tukey's multiple-comparison test.

\section{Results}

\section{Selection and Identification of hRAGE-NIH3T3 Cell Colonies}

Lentivirus is a useful tool in gene delivery and has high efficiency to integrate exogenous gene in the genome of host cells. In this study, pLVX-Puro lentivirus vector was used as a backbone and the insert was a hRAGE-fused HA tag DNA fragment. The expected molecular weight of recombinant hRAGE-HA expressed by NIH3T3 should be approximately $43 \mathrm{kDa}$ and it could be recognized by the anti-HA tag antibody in Western blotting. Interestingly, there were two bands on the membrane detected by the anti-HA antibody with molecular weights of approximately 45 and $55 \mathrm{kDa}$, respectively (-Fig. 2 ). The migration pattern of hRAGE-HA was in accordance with literature data. ${ }^{26}$

Colony selection played a critical role in the construction of transgenic cells for stable expression of exogenous genes because protein levels of every cell are distinctive in the cell pool. Cell clones expressing the highest level of hRAGE-HA could be chosen and used in subsequent research studies. We isolated 12 hRAGE cell clones because of their growth capacity in culture. The selected clones were plated on sixwell plates and samples of the cells were collected for Western blotting analysis. Our data showed that three clones have higher hRAGE levels than others (-Fig. 3). These three cell clones, named RAGE-1, RAGE-7, and RAGE-10, were

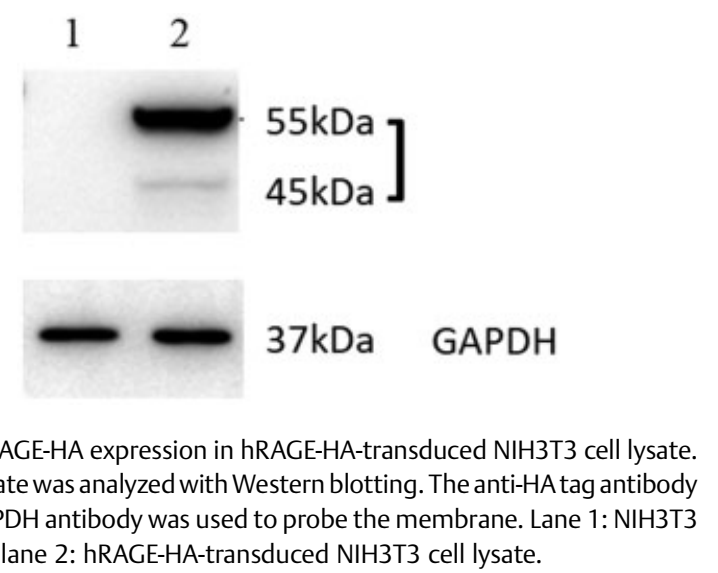

Fig. 2 hRAGE-HA expression in hRAGE-HA-transduced NIH3T3 cell lysate. The cell lysate was analyzed with Western blotting. The anti-HA tag antibody or anti-GAPDH antibody was used to probe the membrane. Lane 1: NIH3T3 cell lysate; lane 2: hRAGE-HA-transduced NIH3T3 cell lysate.

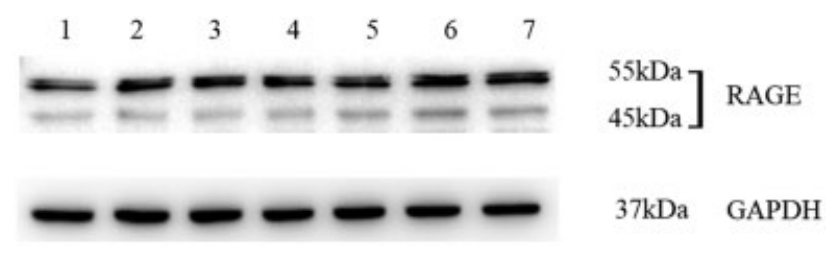

Fig. 3 Expression of hRAGE-HA in different colony samples from colony 1 to 12 . The anti-HA tag antibody or anti-GAPDH antibody was used to probe the membrane.

selected for cell-line establishment, in vitro antibody binding, and ligand bioactivity assays.

\section{hRAGE-NIH3T3 Cell Stability Assay}

The exogenous genes in host cells could be inactive due to genome epigenetic modifications (reference: doi 10.1074/jbc. M109.092007). To investigate the stability of hRAGE-HA integrating in NIH3T3 cells, hRAGE-HA expression in selected clones was analyzed from the 1 st to the 20th generation. We found that the levels of hRAGE-HA were not significantly different during 20 generation passages. This indicated that the hRAGE-HA was stably expressed in hRAGE-NIH3T3 cells (-Fig. 4).

\section{Anti-hRAGE Antibody Recognition of hRAGE-HA in Engineered Cells}

Recently, over 30 therapeutic antibodies have been used in the clinic and target receptor-overproducing cell lines were involved in all stages of therapeutic antibody development, for example, cell-based binding and biological function assays. ${ }^{27,28}$ Anti-hRAGE rabbit polyclonal antibody was used to verify whether hRAGE-HA localized on the cell surface could be specifically recognized by anti-hRAGE antibody. Based on the assay of FACS, we found that signals from both anti-hRAGE antibody and control IgG were very weak on NIH3T3 cells. The data showed that anti-hRAGE antibody had very low affinity in binding to mouse RAGE on the surface of NIH3T3 cells (-Fig. 5A). Meanwhile, in hRAGE-NIH3T3 cells, signals of anti-hRAGE were stronger than that of control IgG ( - Fig. 5B).

\section{Anti-hRAGE Antibody Protection of hRAGE-NIH3T3 Cells against $A \beta$-Induced Apoptosis}

It is well known that therapeutic antibody works as a receptor inhibitor depending on distribution of ligands bound to receptor. We investigated hRAGE-HA biological function induced by $A \beta$, which is one of the RAGE ligands and could induce cell apoptosis. ${ }^{29}$ To confirm the availability

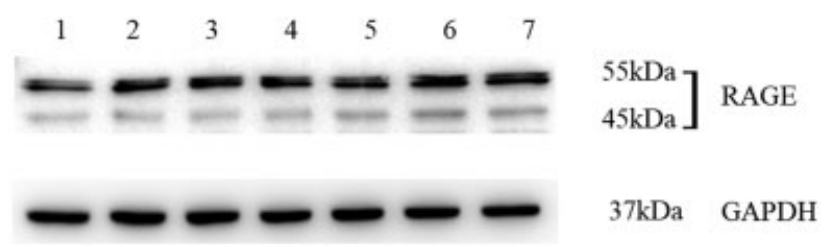

Fig. 4 The hRAGE-HA stably expresses through 20 generations in hRAGENIH3T3. The samples were sequentially collected at generations $1,4,7,10$, 13,16 , and 20. These sample were loaded on SDS-PAGE from lane 1 to lane 7 , respectively. The anti-HA tag antibody or anti-GAPDH antibody was used to probe the membrane in Western blotting assay. 
A

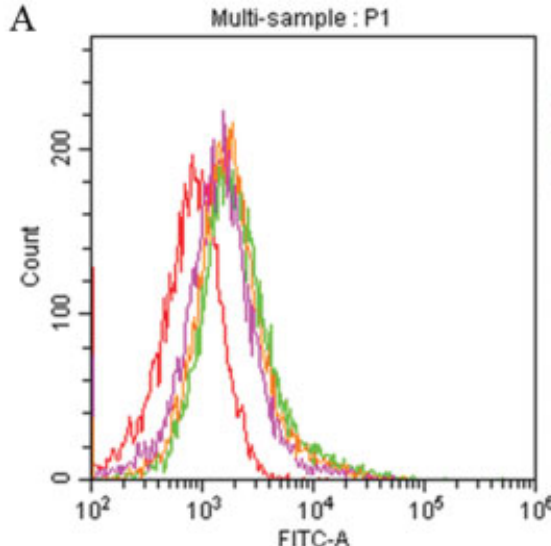

B

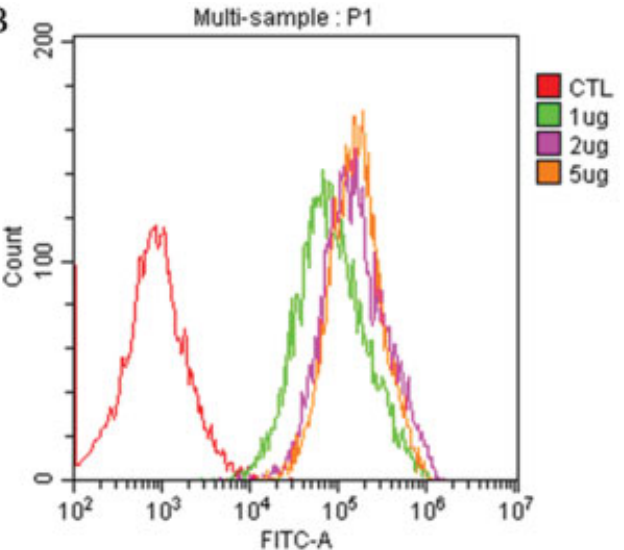

Fig. 5 Anti-hRAGE antibody had high affinity binding with hRAGE-NIH3T3 cells (A: NIH3T3 cells, B: hRAGE-NIH3T3 cells).
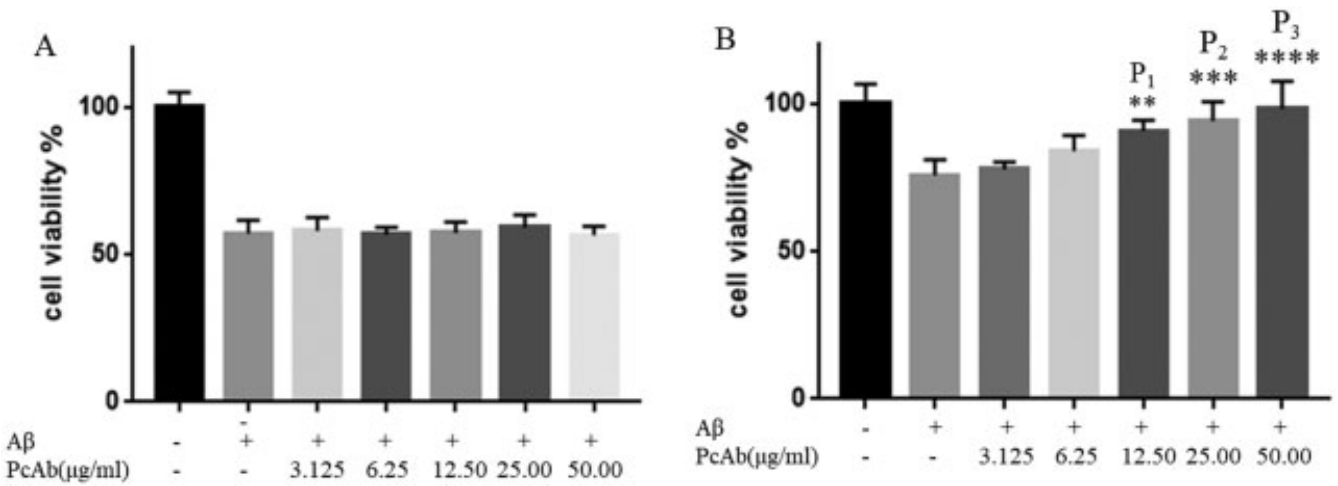

Fig. 6 Anti-hRAGE antibody improvement of hRAGE-NIH3T3 cell survival following A treatment. The anti-hRAGE rabbit polyclonal antibody (PcAb) and amyloid $\beta(A \beta)$ were used to treat NIH3T3 cells $(A)$ or hRAGE-NIH3T3 cells $(B)$. Data of cell viability were normalized by the control group. Experiments were repeated three times: $p_{1}$ value $=0.0017, p_{2}$ value $=0.0001, p_{3}$ value $=0.0001$.

of hRAGE-NIH3T3 cells for anti-RAGE molecule screening and functional study, NIH3T3 cells or hRAGE-NIH3T3 cells were treated with $A B$ or $A \beta /$ anti-hRAGE antibodies for 48 hours. The data showed that anti-hRAGE antibodies significantly improved hRAGE-NIH3T3 survival under A $\beta$ stimulation, but the antibody had no effects on protecting NIH3T3 cells ( - Fig. 6). hRAGE-HA in hRAGE-NIH3T3 cells also had biological function and the engineered cell line could be used to access biological activities of anti-hRAGE antibodies or ligands in vitro.

\section{Discussion}

Pulmonary fibrosis, diabetes complications, and other diseases are closely associated with overactivation of the RAGE pathway. RAGE inhibitors have the potential to treat these diseases. ${ }^{30,31}$ We have analyzed RAGE expression in several human cancer cell lines by Western blotting and FACS. The protein level of RAGE in those tumor cells was much lower than that in hRAGE-NIH3T3 cells (data not shown). The hRAGE-NIH3T3 cell line should have good sensitivity and high efficiency for screening antibodies or interaction partners of hRAGE. We employed a lentivirus gene delivery technique to construct engineered cell lines because the lentivirus system has high safety and efficiency in transfer- ring exogenous genes into host cells. ${ }^{32}$ It is also used to produce chimeric antigen T-cells for clinical treatment. ${ }^{33}$

In summary, the hRAGE-transgenic NIH3T3 cell line established in this study stably expresses hRAGE through 20 generations and it was successfully applied to analyze specific affinity and biological activity of anti-RAGE antibody. This cell line may be a powerful tool for the development of drugs targeting the RAGE pathway in the future. It could also be used to study the molecular mechanism of RAGE-mediated signal transduction in vitro.

\section{Funding}

This research work was supported by the National Natural Science Foundation of China (31671388 and 81302825), the National Science and Technology Major Project "Key New Drug Creation and Manufacturing Program" (2019ZX09201001), and the Shanghai Jiao Tong University Medical-Engineering Joint Project (YG2019QNA50).

Conflict of Interest

The authors declare no conflicts of interest.

\section{Acknowledgment}

We thank Ms. Aerzuguli Maimaiti for helping in preparing some solutions and for performing ELISA assay. 


\section{Reference}

1 Rouhiainen A, Kuja-Panula J, Tumova S, Rauvala H. RAGE-mediated cell signaling. Methods Mol Biol 2013;963:239-263

2 Fritz G. RAGE: a single receptor fits multiple ligands. Trends Biochem Sci 2011;36(12):625-632

3 Yamagishi S, Matsui T. Soluble form of a receptor for advanced glycation end products (sRAGE) as a biomarker. Front Biosci (Elite Ed) 2010;2(04):1184-1195

4 Han SH, Kim YH, Mook-Jung I. RAGE: the beneficial and deleterious effects by diverse mechanisms of actions. Mol Cells 2011;31 (02):91-97

5 Huttunen HJ, Fages C, Rauvala H. Receptor for advanced glycation end products (RAGE)-mediated neurite outgrowth and activation of NF-kappaB require the cytoplasmic domain of the receptor but different downstream signaling pathways. J Biol Chem 1999;274 (28):19919-19924

6 Fages C, Nolo R, Huttunen HJ, Eskelinen E, Rauvala H. Regulation of cell migration by amphoterin. J Cell Sci 2000;113(Pt 4):611-620

7 Yan SD, Chen X, Fu J, et al. RAGE and amyloid-beta peptide neurotoxicity in Alzheimer's disease. Nature 1996;382(6593):685-691

8 Hofmann MA, Drury S, Fu C, et al. RAGE mediates a novel proinflammatory axis: a central cell surface receptor for S100/ calgranulin polypeptides. Cell 1999;97(07):889-901

9 Ray R, Juranek JK, Rai V. RAGE axis in neuroinflammation, neurodegeneration and its emerging role in the pathogenesis of amyotrophic lateral sclerosis. Neurosci Biobehav Rev 2016;62:48-55

10 Deane R, Singh I, Sagare AP, et al. A multimodal RAGE-specific inhibitor reduces amyloid $\beta$-mediated brain disorder in a mouse model of Alzheimer disease. J Clin Invest 2012;122(04):1377-1392

$11 \mathrm{Li} \mathrm{Y,} \mathrm{Wu} \mathrm{R,} \mathrm{Tian} \mathrm{Y,} \mathrm{et} \mathrm{al.} \mathrm{RAGE/NF-kB} \mathrm{signaling} \mathrm{mediates} \mathrm{lipopoly-}$ saccharide induced acute lung injury in neonate rat model. Int J Clin Exp Med 2015;8(08):13371-13376

12 Liu Y, Liang C, Liu X, et al. AGEs increased migration and inflammatory responses of adventitial fibroblasts via RAGE, MAPK and NFkappaB pathways. Atherosclerosis 2010;208(01):34-42

13 Yeh CH, Sturgis L, Haidacher J, et al. Requirement for p38 and p44/ p42 mitogen-activated protein kinases in RAGE-mediated nuclear factor-kappaB transcriptional activation and cytokine secretion. Diabetes 2001;50(06):1495-1504

14 Bianchi R, Giambanco I, Donato R. S100B/RAGE-dependent activation of microglia via NF-kappaB and AP-1 Co-regulation of COX2 expression by S100B, IL-1beta and TNF-alpha. Neurobiol Aging 2010;31(04):665-677

15 Perkins ND. Integrating cell-signalling pathways with NF-kappaB and IKK function. Nat Rev Mol Cell Biol 2007;8(01):49-62

16 Tanji N, Markowitz GS, Fu C, et al. Expression of advanced glycation end products and their cellular receptor RAGE in diabetic nephropathy and nondiabetic renal disease. J Am Soc Nephrol 2000;11(09):1656-1666

17 Wendt T, Harja E, Bucciarelli L, et al. RAGE modulates vascular inflammation and atherosclerosis in a murine model of type 2 diabetes. Atherosclerosis 2006;185(01):70-77
18 Onyeagucha BC, Mercado-Pimentel ME, Hutchison J, Flemington EK, Nelson MA. S100P/RAGE signaling regulates microRNA-155 expression via AP-1 activation in colon cancer. Exp Cell Res 2013; 319(13):2081-2090

19 Tesarova P, Cabinakova M, Mikulova V, Zima T, Kalousova M. RAGE and its ligands in cancer - culprits, biomarkers, or therapeutic targets? Neoplasma 2015;62(03):353-364

20 Xia P, He H, Kristine MS, et al. Therapeutic effects of recombinant human S100A6 and soluble receptor for advanced glycation end products(sRAGE) on $\mathrm{CCl}_{4}$-induced liver fibrosis in mice. Eur $\mathrm{J}$ Pharmacol 2018;833:86-93

21 Pontén F, Jirström K, Uhlen M. The Human Protein Atlas-a tool for pathology. J Pathol 2008;216(04):387-393

22 Shafaghat F, Abbasi-Kenarsari H, Majidi J, Movassaghpour AA, Shanehbandi D, Kazemi T. preparation of proper immunogen by cloning and stable expression of cDNA coding for human hematopoietic stem cell marker CD34 in NIH-3T3 mouse fibroblast cell line. Adv Pharm Bull 2015;5(01):69-75

23 Salehi-Lalemarzi H, Shanehbandi D, Shafaghat F, et al. Cloning and stable expression of cDNA coding for platelet endothelial cell adhesion molecule-1 (PECAM-1, CD31) in NIH-3T3 cell line. Adv Pharm Bull 2015;5(02):247-253

24 Abbasi-Kenarsari H, Shafaghat F, Baradaran B, Movassaghpour AA, Shanehbandi D, Kazemi T. Cloning and expression of CD19, a human B-cell marker in NIH-3T3 cell line. Avicenna J Med Biotechnol 2015;7(01):39-44

25 Weng S, Zhou L, Deng Q et al. Niclosamide induced cell apoptosis via upregulation of ATF3 and activation of PERK in Hepatocellular carcinoma cells. BMC Gastroenterol 2016;16:25

26 Hudson BI, Carter AM, Harja E, et al. Identification, classification, and expression of RAGE gene splice variants. FASEB J 2008;22(05): 1572-1580

27 Liu Z, O'Rourke J. Expediting antibody discovery with a cell and bead multiplexed competition assay. SLAS Discov 2018;23(07): 667-675

28 Elgundi Z, Reslan M, Cruz E, Sifniotis V, Kayser V. The state-of-play and future of antibody therapeutics. Adv Drug Deliv Rev 2017; 122:2-19

29 Kim Y, Mook-Jung I. PRAK mediates A $\beta$-RAGE driven autophagy pathway. Oncotarget 2017;8(04):5648-5649

30 Oczypok EA, Perkins TN, Oury TD. All the "RAGE" in lung disease: the receptor for advanced glycation endproducts (RAGE) is a major mediator of pulmonary inflammatory responses. Paediatr Respir Rev 2017;23:40-49

31 Litwinoff E, Hurtado Del Pozo C, Ramasamy R, Schmidt AM. Emerging targets for therapeutic development in diabetes and its complications: the RAGE signaling pathway. Clin Pharmacol Ther 2015;98(02):135-144

32 Sakuma T, Barry MA, Ikeda Y. Lentiviral vectors: basic to translational. Biochem J 2012;443(03):603-618

33 Milone MC, O'Doherty U. Clinical use of lentiviral vectors. Leukemia 2018;32(07):1529-1541 